\title{
Structural Identification of Static Systems with Distributed Lags
}

\author{
Nikolay Karabutov \\ Department of Problems Control, Moscow state engineering university of radio engineering, electronics and automation, 119454, Moscow, \\ Russia
}

\begin{abstract}
The approach to structural identification of static systems with the distributed lags is offered. The criterion of an estimation of linearity of system in parametrical space is introduced. The criterion is based on construction of set of secants for system. The special space for an estimation of structural parameters of system is selected. The concept of level of nonlinearity of system is introduced and the method of h is estimation is reduced. The analogue of criterion of Darbin-Watson is reduced. Criteria of decision-making in the presence of a lag as in an output variable, and input variables are offered. It is shown that as magnitude of a lag performance on an output variable one can to use an estimation of parameters of coefficient of structural properties on the specified variable. Estimations of parameters of coefficient of structural properties are based on an evaluation of Lyapunov characteristic indicators.
\end{abstract}

Keywords Structural identification, Distributed lags, Linearity level, Secant

\section{Introduction}

With the distributed delays the set of publications is devoted problems of identification of static plants. Such models are widely applied in econometrics and economy[1-4], to the technician [5-7], medicine[8-10]. Delay can have both independent, and dependent variables. The account of the distributed lags leads to autocorrelation between variables $[1,2,4]$. Autocorrelation hampers identification of parameters of plant. Various models of approximation of parameters are applied to a abatement of influence of autocorrelation on identification process at the distributed lags. It allows to reduce number of estimated parameters of plant. The Koyck scheme[2,12], based on a change of factors of model on a decreasing geometrical progression is most widely applied. The model I. Fisher[1, 11] is based on a change of factors of model on the set decreasing arithmetical progression. Arithmetical and geometrical models are applied in that case when plant parameters decrease from the first me mbers of a progression. Considering it, S. Almon [13] has added more flexibilities of model of I. Fisher, having applied polynomial the law of a modification of factors. In[11] the law of a change of factors is offered at the distributed lags according to logarithmic normal distribution. The same idea based on application the exponential law of distribution, is considered in[14]. In[15] Pascal distribution which leads to the simple form for factors

* Corresponding author:

kn22@yandex,ru (Nikolay Karabutov)

Published online at http://journal.sapub.org/control

Copyright (C) 2012 Scientific \& Academic Publishing. All Rights Reserved is applied.

The a priori representation of correlation between the distributed lags in the form of some rational polynomial is considered in[16]. Other approaches to description of factors at the distributed lags are studied in[4].

The considered models of factors minimise number of unknown parameters. To an estimation of parameters apply a method least-squares method or its modifications [1-4]. In these works the model structure is postulated a priori and the problem of parametrical identification is considered. In[17] the interactive algorith $m$ of an estimation of parameters of static plant with the distributed lags is offered. The length of a lag is set and does not become any suppositions about correlation between plant parameters. The case of a piecewise monotonic change of parameters of plant is considered. In a number of works methods of a choice of maximu m length of a lag are offered. At the heart of applied approaches the statistics which are based on the analys is of residuals [1,15,18] lie. In[19, 20] various methods of an estimation of parameters of model with the distributed lag in a case of a priori set structure of model are considered. In[21] influence of the a priori information received from the analysis of the empirical data, on a choice of structure of model with the distributed lag on an output variable is analyzed. In work[22] are described algorithm of an estimation of parameters of model with the distributed lag. On the basis of results of modelling the structure of model which explains a mis match of model applied now in the American interest rate on federal funds is selected. $\operatorname{In}[23]$ process of inflation with the help autoregressive models is researched. The choice of length of a lag is carried out on the basis of consecutive magnification of delays and an 
estimation of adequacy of the received model. Then the criterion of Akaike and Bayesian Information Criteria are applied and the solution is made on model structure. The case of a priori uncertainty concerning structure and plant parameters was not study.

In the given work the approach to structural identification of static plants in the conditions of uncertainty is offered. It is based on application of the static structures describing properties of plant[24] in special space. The criterion of linearity (autocorrelation) of variables of plant is introduced. Algorith ms of decision-making on maximum length of the distributed lag are offered. They do not demand an evaluation of the statistics. The analogue of criterion Darbina-Watson criterion for a considered case is reduced.

2. Problem Statement

Consider plant

$$
y_{n}=A^{T} U_{n}+B^{T} X_{n}+\xi_{n}
$$

where $y_{n} \in R$ is an exit, $U_{n} \in R^{k}$ is the input vector which elements are limited, is limiting nondegenerate functions, $X_{n}=X\left(u_{i, n} \in U_{n}\right) \in R^{m}$ is a vector of the distributed lags on $\quad u_{i, n} \in U_{n} \quad, \quad X_{n}=\left[u_{i, n-1}, u_{i, n-2}, \ldots, u_{i, n-m}\right]^{T}$, $n \in J_{N}=[0, N]$ is discrete time, $N<\infty, \quad A \in R^{k}, B \in R^{m}$ are vectors of constant parameters, $\xi_{n} \in R$ is a perturbation, $\left|\xi_{n}\right|<\infty$ for all $n \in J_{N}$.

Consider that general case $\xi_{n} \in R$ and $u_{i, n} \in U_{n}$ $(i=\overline{1, N})$ are irregular functions of time.

For (1) the set of the measured values is known

$$
\mathrm{I}_{o}=\left\{y_{n}, U_{n} \forall n \in J_{N}\right\}
$$

and map $\Gamma_{o}:\left\{U_{n}\right\} \times\left\{y_{n}\right\} \quad \forall n \in J_{N}$ describing an observable informational portrait $[7,24]$.

It is necessary $\mathrm{I}_{o}, \Gamma_{o}$ to estimate on the basis of analysis structure of plant (1). It means that it is necessary to estimate degree of linearity and dimension of vector $X_{n}$.

3. An Estimation of Level of Linearity of System(1)

Consider a contraction of observable informational portrait $\left.\Gamma_{o}^{u_{i}} \subset \Gamma_{o}\right|_{u_{i} \in U} \quad \forall i=\overline{1, k}$ and for everyone

$\Gamma_{o}^{u_{i}}$ construct a secant

$$
\bar{\gamma}\left(y, u_{i}\right)=a_{i} u_{i, n}
$$

where $a_{i}$ are some real numbers.

Introduce set on (2) set of secants for $\Gamma_{o}$

$$
\mathrm{S}(U, y)=\left\{\bar{\gamma}\left(y, u_{i}\right) \quad \forall i=\overline{1, k}\right\}
$$

Definition 1[24]. Field of structures $S_{S}$ of system (1) name set of maps $\bar{\gamma}\left(y, u_{i}\right) \subset\left\{u_{i}\right\} \times\{y\} \quad \forall i=\overline{1, k} \quad$ on Euclidean plane $\mathrm{E}$

$$
S_{S}=\mathrm{S}(U, y)
$$

Designate

$\Phi_{n}=\left[\bar{\gamma}\left(y_{n}, u_{1, n}\right), \bar{\gamma}\left(y_{n}, u_{2, n}\right), \ldots, \bar{\gamma}\left(y_{n}, u_{k, n}\right)\right]^{T}$ and consider the equation

$$
\hat{y}_{n}=\Psi^{T} \Phi_{n}
$$

where vector $\Psi \in R^{k}$ define by means of a least-squares method. Estimation $\Psi$ exists on the basis of the suppositions made in section 2 concerning input $U_{n}$.

Completeness of system (1) in the field of structures $S_{S}$ estimate on the basis of the following statement[24].

The orem 1. Consider a vector of informative variables $U_{n} \in R^{k}$ and a field of structures $S_{S} \subset \mathrm{S}(U, y)$ for (1). Then the field of structures $S_{S}$ of system(1) is full, if

$$
\sum_{i=1}^{k} \psi_{i}=1
$$

where $\psi_{i}$ there is $i$-th element of vector $\Psi$ in (4).

The theorem 1 gives linearity sufficient conditions (nonlinearities, collinearity) systems (1) on the set field of structures $S_{S}$. If the condition (4) is fulfilled, that field $S_{S}$ is full. Hence $S_{S}$ is a linear span of an exit of system (1). Otherwise make a solution on presence of nonlinearity or collinearity (autocorrelation) in system (1).

Let

$$
\sum_{i=1}^{k} \psi_{i}=\chi
$$

Magnitude $\Delta(\Psi)=\chi-1$ name level of nonlinearity of system (1) in parametrical space $\mathscr{P}_{\mathscr{P}}=(A, B)$. As nonlinearities and lags lead to occurrence multicollinearity in (1) $\Delta(\Psi)$ will accept small values.

\section{Set for an Estimation of Structure of the Distributed Lag of Plant}

Following [7,24], generate au xiliary set for an estimation of structure (1). Introduce variable $s_{n}=I^{T} U_{n}$, where $I \in R^{k}$ is an unit vector, and apply model $\hat{y}_{s, n}^{n}=\hat{a}_{s} s_{n}$. Define parameter $\hat{a}_{s} \in R$ from a condition

$$
\arg \min _{\hat{a}_{s}}\left(\hat{y}_{s, n}-y_{n}\right)^{2}=\hat{a}_{s}^{*}
$$

Let $e_{n}=\hat{y}_{s, n}-y_{n}$ is a variable which contains the data about structure of a lag of system (1). As argument $X\left(u_{i, n}\right)$ use variable $u_{i, n} \in U_{n}$ which ensures maximum value of coefficient of determination $r_{e, u_{i}}^{2}$ between $e$ and $u_{i}$. As shown in[24], set $\left\{e_{n}, u_{i, n}\right\}$ does not allow to solve a problem of structural identification. Therefore introduce coefficient of structural properties (CSP)[7] 


$$
k_{e, u_{i}, n}=k_{s}\left(e, u_{i}, n\right)=\frac{e_{n}}{u_{i, n}}
$$

and generate set

$\mathrm{I}_{k}=\mathrm{I}_{k}(k, e)=\left\{e_{n}, k_{n}, n \in J_{N}=[0, N]\right\}, k_{n} \stackrel{\mathrm{df}}{=} k_{e, u_{i}, n}$.

Definition 2. Name $\mathscr{P}_{s}=(k, e)$ structural space of the system(1), allowing to identify structure of vector $X_{n}$.

On $J_{N}$ will order $k_{e, u_{i}, n}$ on increase. Generate $\left\{k_{q}^{v}\right\}$, where $k_{q}^{v}=k_{s}\left(e, u_{i}, q\right), \quad q \in J_{N}^{v}=[0, N]$. As to everyone $k_{q}^{v}$ there corresponds value $e_{q}^{v}$ receive $\mathrm{I}_{k}^{v}=\left\{e_{q}^{v}, k_{q}^{v}, q \in J_{N}^{v}\right\} . \quad$ In $\quad \mathcal{P}_{s}$ define map $\Gamma_{e, k}^{v}:\left\{k_{q}^{v}\right\} \times\left\{e_{q}^{v}\right\}$ and structure $S_{k, e}^{v}$ corresponding to it. Now the problem is shown to an estimation of structure $X_{n}$ on the basis of analysis $\mathrm{I}_{k}^{v}$ and $S_{k, e}^{v}$. Such approach well works at an estimation of structure of nonlinear static systems[24]. For systems with the distributed lag he demands modification.

\section{Decision-Making on Length of a Lag in $X\left(u_{i, n}\right)$}

Set $\mathrm{I}_{e, u}=\left\{e_{n}, u_{i, n}\right\}$ contains uncertainty $\mathcal{N}_{L}$ and $\mathcal{N}_{L g} . \mathcal{N}_{L}$ is an incomplete account of linear making system (1). $\mathcal{N}_{L g}$ is a presence of perturbation from the distributed lag. For elimination $\mathcal{N}_{L}$ construct a secant for $e_{n}$

$$
\bar{\gamma}\left(e, u_{i, n}\right)=\alpha_{0}+\alpha_{1} u_{i, n}
$$

where $\alpha_{0}, \alpha_{1}$ define as a solution of a problem (5).

Introduce new variable $\varepsilon_{n}=e_{n}-\bar{\gamma}\left(e, u_{i}\right)$ which does not contain $\mathcal{N}_{L}$. For estimation $\mathcal{N}_{L g}$ analyse set $\left\{\varepsilon_{n}, u_{i, n}\right\}$.

For deriving of a provisional estimate of maximum lag $m$ fulfil following operations. Set admissible level $\rho>0$ of coefficient of determination $r_{e, u_{i}}^{2}$. Apply the following algorith m.

Algorithm $C D_{\rho}$.

1. Suppose $m=1$.

2. Construct a secant

$$
\bar{\gamma}\left(e, u_{i, n-m}\right)=\bar{\gamma}_{e, k}=\alpha_{0}+\alpha_{1} u_{i, n-m}
$$

and define $r_{e, u_{i, n-m}}^{2}$.

3. Verify up condition $r_{e, u_{i, n-m}}^{2} \geq \rho$.

4. If the condition is fulfilled, suppose $m=m+1$ and go to a step 2 , differently fin ish work.

In work the multiple-functional approach to structural identification is applied. Therefore known methods of a choice of length of the lag, based on statistical criteria (section 1 see), are inapplicable.

Considering it, to an estimation of independence of elements of vector $X_{n}$ apply the theorem 1. As the analysis of set $\mathrm{I}_{e, u}$ is in this case ineffective, that use results of section 4 and generate set

$$
\mathrm{I}_{\varepsilon, k_{x}}=\left\{\varepsilon_{n}, k_{\varepsilon, x_{i, n}}, i=\overline{1, m} \forall n \in J_{N}\right\}
$$

where $k_{\varepsilon, x_{i}, n}$ calculate on the basis of (6), considering $e_{n}=\varepsilon_{n}, x_{i, n} \in X_{n}$.

$$
\begin{aligned}
\text { On } \mathrm{I}_{\varepsilon, k_{X}} \quad \text { will introduce transformation } \\
\\
\Gamma_{\varepsilon, k_{\varepsilon, x_{i}}}:\left\{k_{\varepsilon, x_{i, n}}\right\} \times\left\{\varepsilon_{n}\right\}
\end{aligned}
$$

to which in space $\mathcal{P}_{s, \varepsilon}=\left(k_{\varepsilon, x_{i, n}}, \varepsilon\right)$ there corresponds structure $S_{k \varepsilon, x}, \varepsilon, k_{\varepsilon, x}=k_{\varepsilon, x_{i}}$. Construct secants for $S_{k \varepsilon, x, \varepsilon}$

$$
\bar{\gamma}\left(\varepsilon_{n}, k_{\varepsilon, x_{i, n}}\right)=\beta_{i} k_{\varepsilon, x_{i, n}}, \forall i=\overline{1, m_{*}},
$$

where $\beta_{i}$ are the coefficients defined as a result of a solution of a problem (5), $m_{*}$ is value $m$ received on the basis of application of algorithm $C D_{\rho}$.

Generate a vector

$\Phi_{\varepsilon, n}=\left[\bar{\gamma}\left(\varepsilon_{n}, k_{\varepsilon, x_{1, n}}\right), \bar{\gamma}\left(\varepsilon_{n}, k_{\varepsilon, x_{2, n}}\right), \ldots, \bar{\gamma}\left(\varepsilon_{n}, k_{\varepsilon, x_{m_{*}, n}}\right)\right]^{T(9)}$ and apply model $\hat{\varepsilon}_{n}=\Psi_{\varepsilon}^{T} \Phi_{\varepsilon, n}$ to forecasting of change $\varepsilon_{n}$, where vector $\Psi_{\varepsilon} \in R^{m_{*}}$ is defined on the basis of outcomes of section 3 .

The statement following directly from the theorem 1 is fair.

Theorem 2. Let on set $\mathrm{I} \varepsilon, k_{x}$ the field of secants for $\varepsilon_{n}$ is constructed

$$
S_{\varepsilon}=\left\{\bar{\gamma}\left(\varepsilon_{n}, k_{\varepsilon, x_{i, n}}\right)\left(\forall i=\overline{1, m_{*}}\right) \&\left(n \in J_{N}\right)\right\}
$$

and model $\hat{\varepsilon}_{n}=\Psi_{\varepsilon}^{T} \Phi_{\varepsilon, n}$ is applied to forecasting of a change of variable $\mathcal{E}_{n}$. Then vector $X_{n} \in R^{m_{*}}$ is an ele ment of structure of system(1), if

$$
\sum_{i=1}^{m_{*}} \psi_{\varepsilon, i} \neq 1
$$


where $m_{*}$ it is defined by means of algorithm $C D_{\rho}$, $\psi_{\varepsilon, i} \in \Psi_{\varepsilon}$.

Remark. As $\varepsilon_{n}$ contains the information on influence of vector $X_{n}$ at statistical interpretation $U_{n}, \xi_{n}$ use analag of criterion of criterion of Darbin-Watson

$$
d=\frac{\sum_{n=1}^{N} \varepsilon_{n}^{2}}{\sum_{n=1}^{N} e_{n}^{2}}
$$

Level of nonlinearity of the system (1), generated by correlation of elements of vector $X_{n}$ is equal

$$
\Delta\left(\Psi_{\varepsilon}\right)=\sum_{i=1}^{m_{*}} \psi_{\varepsilon, i}-1
$$

Theorem 3. Consider the set of secants $S_{\varepsilon}$ set on $\mathrm{I}_{\varepsilon, k_{x}}$ and secant $\bar{\gamma}\left(e, k_{e, u_{i}}\right)=\alpha_{1} k_{e, u_{i}}$ for $S_{k e, u_{i}}, e$. Let for them coefficients of determination $r_{\varepsilon, k \varepsilon, x_{z}}^{2}, \quad z=\overline{1, m}$ and $r_{e, k_{e}, u_{i}}^{2}$ are known. Then vector $X\left(u_{i, n}\right) \in R^{m}$ is an element of structure of $\operatorname{system}(1)$, if $\forall z=\overline{1, m}$

$$
\left|r_{\varepsilon, k_{\varepsilon, x_{z}}^{2}}^{2}-r_{e, k_{e, u_{i}}}^{2}\right| \leq \delta_{x}
$$

where $\delta_{x} \geq 0$ is a specified magnitude.

At structure $S_{k, e}^{v}$ analysis, the distributed lag of system (1) interpret as a nonlinear component (1). Therefore design the corresponding procedure, allowing to make the solution on a class of uncertainty $\mathcal{N}_{L g}$. Apply the following approach.

Consider set $\mathrm{I}_{k}^{v}$ and static structure $S_{k, e}^{v}$ corresponding to it. Construct the secants $S_{k, e}^{v}$

$$
\begin{gathered}
\bar{\gamma}_{1}\left(e_{q}^{v}, k_{q}^{v}\right)=a_{1}^{v} k_{q}^{v} \\
\bar{\gamma}_{2}\left(e_{q}^{v}, k_{q}^{v}\right)=a_{2,0}^{v} k_{q}^{v}+a_{2,1}^{v}\left(k_{q}^{v}\right)^{2}
\end{gathered}
$$

and define for them coefficients of determination $r_{1}^{2}$, $r_{2}^{2}$.

Consider CSP

$$
k_{e, u_{i}, n, \pi}=k_{s, \pi}=\frac{e_{n}}{u_{i, n}^{\pi}}, \pi<\infty
$$

and map $\Gamma_{e, k, \pi}^{v}:\left\{k_{s, \pi, q}^{v}\right\} \times\left\{e_{q}^{v}\right\}, q \in J_{N}^{v}$ to which there corresponds structure $S_{k_{s, \pi}, e}^{V}$. Construct for $S_{k_{s, \pi}, e}^{V}$ a secant

$$
\bar{\gamma}\left(e_{q}^{v}, k_{s, \pi, q}^{v}\right)=\bar{\gamma}_{\pi}=a_{\pi}^{v} k_{s, \pi, q}^{v}
$$

and parameter $\pi$ select so that the factor of determination $r_{\bar{\gamma}}^{2}$ for (11) belonged to interval $\left[r_{1}^{2}, r_{2}^{2}\right]$.

Further consider CSP

$$
k_{e, x, n}=k_{s, x}=\frac{e_{n}}{I^{T} X_{n}}
$$

where $I \in R^{m}$ is an unit vector. Construct a secant for $S_{s_{s, x}, e}^{v}$

$$
\bar{\gamma}\left(e_{q}^{v}, k_{s, x, q}^{v}\right)=\bar{\gamma}_{x}=a_{\pi}^{v} k_{s, x, q}^{v}
$$

Designate coefficient of determination for $\bar{\gamma}_{x}$ through $r_{\gamma_{x}}^{2}$.

Theorem 4. Consider structures $S_{k_{s, \pi}, e}^{v}, S_{k_{s, x}, e}^{v}$ and secants (11), (12) corresponding to them. If for structure $S_{k_{s, \pi}, e}^{v}, S_{k_{s, x}, e}^{v}$ secants the condition is satisfied

$$
r_{e, k_{x}}^{2} \geq r_{e, k_{s, \pi}}^{2}
$$

then $X_{n}$ is an element of structure of system(1).

The proof of the theorem 4 is obvious. It is based that structures $S_{k_{s, \pi}, e}^{v}, S_{k_{s, x}, e}^{v}$ describe a change of same variable $e_{q}^{v}$. Therefore a solution about inclusion in structure of system(1) accept concerning that variable $X_{n}$, $u_{i, n}^{\pi}$ which ensures greater coefficient of determination.

Statement. If coefficient of determination $r_{\varepsilon, u_{i}}^{2} \approx 0$, then $r_{\varepsilon, k s, u_{i}}^{2} \approx 1$.

Proof. From $r_{\varepsilon, u_{i}}^{2} \approx 0$ follows that parameter $\hat{a}$ of model $\hat{\varepsilon}_{n}=\hat{a} u_{i, n}$ predicting a change of variable $\varepsilon_{n}$, is equal to zero. Then for $r_{\varepsilon, k_{s, u_{i}}}^{2}$ receive

$$
r_{\varepsilon, k_{s, u_{i}}}^{2}=1-\frac{\sum_{n=1}^{N}\left(\hat{\alpha} k_{s, u_{i, n}}-\varepsilon_{n}\right)^{2}}{\sum_{n=1}^{N} \varepsilon_{n}^{2}}
$$

where $k_{s, u_{i, n}}=\varepsilon_{n} / u_{i, n}$. Substituting $k_{s, u_{i, n}}$ in (13), for fraction numerator receive

$$
\sum_{n=1}^{N}\left(\hat{\alpha} k_{s, u_{i, n}}-\varepsilon_{n}\right)^{2}=\sum_{n=1}^{N} \frac{\varepsilon_{n}^{2}}{u_{i, n}^{2}}\left(\hat{\alpha}-u_{i, n}\right)^{2}
$$


If $\hat{\alpha}=\bar{u}_{i, n}$ where $\bar{u}_{i, n}$ there is average value $u_{i, n}$, then $r_{\varepsilon, k s, u_{i}}^{2} \approx 1$.

Results of modelling confirm the made statement.

Consider a case when vector $X_{n}$ in (1) contains the distributed lags on $y_{n}$, that is $X_{n}=X\left(y_{n}\right)$.

\section{Decision-Making on Length of a Lag for $X\left(y_{n}\right)$}

Let $X_{n}=\left[x_{1, n}, x_{1, n}, \ldots, x_{l, n}\right]^{T}$, where $x_{i, n}=y_{n-i}$, $i=\overline{1, l}$. To tentative estimation $l$ apply algorithm $C D_{\rho}$. Decision-making in space $\mathcal{P}_{s}=\left(k_{\varepsilon, x_{i, n}}, e_{n}\right)$, can appear ineffective because of connections between $x_{i, n}$. Therefore consider space $\mathscr{P}_{s, X}=(X, e)$ set on set $\mathrm{I}_{e, X}=\left\{X_{n}, e_{n} \forall n \in J_{N}\right\}$. Consider structure $S_{X, e}$ and its projections $S_{x_{i}, e}$ to plane $\left(x_{i}, e\right)$. For everyone $S_{x_{i}, e}$ define secants $\bar{\gamma}\left(e, x_{i}\right)=\alpha_{0, i}+\alpha_{1, i} x_{i, n}$ $i=\overline{1, l}$ Also generate a vector

$$
\Phi_{e, n}=\left[\bar{\gamma}\left(e_{n}, x_{1, n}\right), \bar{\gamma}\left(e_{n}, x_{2, n}\right), \ldots, \bar{\gamma}\left(e_{n}, x_{l, n}\right)\right]^{T}
$$

Theorem 5. Let on set $\mathrm{I}_{e, X}$ the field of secants for $e_{n}$ is constructed

$$
S_{e}=\left\{\bar{\gamma}\left(e_{n}, x_{i, n}\right) \quad(\forall i=\overline{1, l}) \&\left(n \in J_{N}\right)\right\}
$$

and model $\hat{e}_{n}=\Psi_{e}^{T} \Phi_{e, n}$ is applied to forecasting of variable $e_{n}$. Then vector $X_{n} \in R^{l}$ is an element of structure of system (1), if

$$
\sum_{i=1}^{l} \psi_{e, i} \neq 1
$$

where $l$ define by means of algorithm $C D_{\rho}, \psi_{e, i} \in \Psi_{e}$.

The theorem 5 is analogue of the theorem 2. Performance (16) specifies on presence of dependence between $x_{i, n}$. At statistical interpretation of a problem (16) speaks about autocorrelation of residuals[1]. Level of nonlinearity of system (1) estimate by means of $\Delta\left(\Psi_{e}\right)$.

For an estimation of existence of a lag on $y_{n}$ analyse a change CSP

$$
k_{s}\left(e, x_{i}, n\right)=k_{s, x_{i}, e, n}=\frac{e_{n}}{x_{i, n}}
$$

As the $\log$ on $y_{n}$ is considered, then

$$
k_{s, x_{i}, e, n}=e^{\lambda n \tau}
$$

where $\lambda$ is an eigenvalue of system (1) with $X_{n}=X\left(y_{n}\right), \tau$ is an interval of measurement of the data.

The problem consists in estimation $k_{s}\left(e, x_{i}, n\right)$ on the basis of analysis $\mathbf{I}_{e, x_{i}}$ and identification of parameter $\lambda$ on set $\left\{k_{s}\left(e, x_{i}, n\right), n \tau\right\}$. To estimation $\lambda$ apply Lyapunov characteristic indicators.

Apply model $\hat{e}_{n}=\beta x_{i, n}$ to forecasting of change $e_{n}$ and define parameter $\beta$ by means of a least-squares method. To description $e_{n}$ also apply model $\hat{e}_{n}=\hat{\alpha} k_{s, x_{i}, e, \text { where }}$ $\hat{\alpha}=\bar{x}_{i, n}$. From comparis on of these two models receive

$$
k_{s, x_{i}, e, n}=\beta \frac{x_{i, n}}{\bar{x}_{i, n}}, \forall n \in J_{N}
$$

Arrange values $k_{S, x_{i}, e, n}$ in ascending order and receive set $\mathrm{I}_{k_{x, e}}^{v}=\left\{k_{s, x_{i}, e, q}^{v}, q \in J_{N}\right\}$. Define for $k_{s, x_{i}, e, q}^{v}$ of Lyapunov characteristic indicator[25]

$$
\mu\left[k_{s, x_{i}, e, q}^{v}\right]=\varlimsup_{t_{q} \rightarrow \infty} \frac{1}{t_{q}} \ln \left|\frac{k_{s, x_{i}, e, t_{q}}^{v}}{\max _{t_{q}} k_{s, x_{i}, e, t_{q}}^{v}}\right|
$$

where $\overline{\lim }_{t q \rightarrow \infty}$ is an limit superior, $t_{q}=q \tau$.

Suppose $\hat{\lambda}=\mu$ where $\hat{\lambda}$ there is estimation $\lambda$. To an improving of received estimation $\lambda$ apply the approach offered in[24].

Remark. Estimation $\lambda$ serves as the indicator of presence at system (1) distributed lags on $y_{n}$. Exact estimation $\lambda$ define only at a step of parametrical identification.

Consider the more general case when vector $X_{n}$ in (1) is equal $X\left(y_{n}, u_{i, n}\right)=\left[X_{y, n}^{T}, X_{u, n}^{T}\right]^{T}$, where $X_{y, n}=X\left(y_{n}\right)$, $X_{u, n}=X\left(u_{i, n}\right)$.

\section{Decision-Making on Length of a Lag for $X\left(y_{n}, u_{i, n}\right)$}

Spread the approach stated above to system (1) with $X_{n}=X\left(y_{n}, u_{i, n}\right)$. The provisional estimate of level of linearity of system (1) receive by means of the theorem 1. Introduce variable $e_{n} \in R$ which depends on uncertainty $\mathcal{N} L$. Apply algorithm $C D_{\rho}$ to definition of dimension of vectors $X_{y, n}, X_{u, n}$. For elimination of influence $\mathcal{N}_{L}$ 
construct a secant for $e_{n}$

$$
\bar{\gamma}\left(e, u_{i}, y\right)=\alpha_{0}+\alpha_{1} u_{i, n}+\alpha_{2} y_{n}
$$

where $\alpha_{0}, \alpha_{1} \cdot \alpha_{2}$ define as a solution of a problem (5).

Introduce new variable $\varepsilon_{n}=e_{n}-\bar{\gamma}\left(e, u_{i}, y\right)$ which does not contain $\mathcal{N}_{L}$. To decision-making on lag presence apply results of sections 5,6 .

\section{Examples}

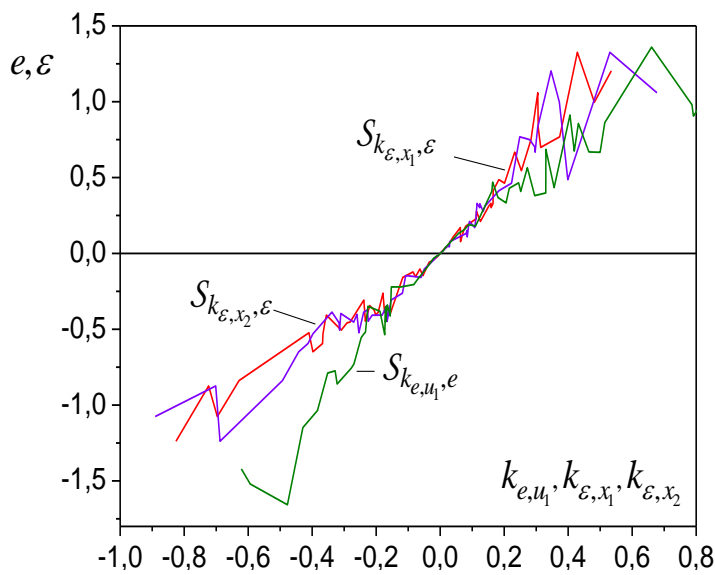

Figure 1. Structures for decision-making on length of lag $X\left(u_{1, n}\right)$

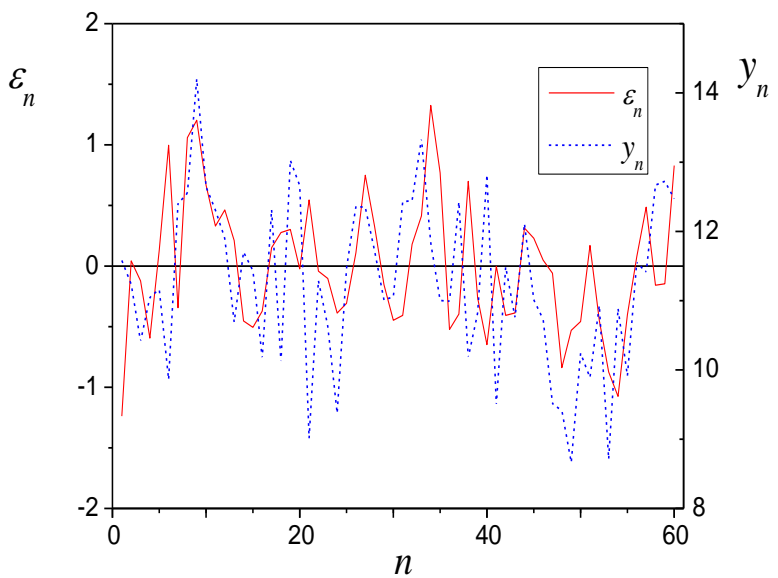

Figure 2. Variables $y_{n}, \varepsilon_{n}$

Consider system (1) with $A=[1.5 ; 2 ; 2.8]^{T}$, $B=[0.7 ; 0.6]^{T}, \quad X_{n} \in R^{2}, \quad X_{n}=X\left(u_{1, n}\right) . \quad u_{i, n} \in U_{n}$ are limited random functions, $\xi_{n}$ is an stochastic variable with zero expectation and a final variance, $\left|\xi_{n}\right| \leq 0.3$. Application of the theorem 1 has shown that the system is not linear, $\Delta(\Psi)=0.03$. Receive set

$$
\mathrm{I}_{e, u}=\left\{e_{n}, u_{i, n}(\forall i=\overline{1,3}) \&\left(\forall n \in J_{N}\right)\right\}
$$

Analysis $\mathrm{I}_{e, u}$ has shown that variable $u_{1, n}$ has a lag. Apply algorithm $C D_{\rho}$ and the theorem 2. The length of a lag on $u_{1, n}$ is equal 2. Indicator $\Delta\left(\Psi_{\varepsilon}\right)$ practically coincides with $\Delta(\Psi)$. To an correction of the received estimation of length of a lag apply the theorem 3 with $\delta_{x}=0.01 \quad \cdot \quad r_{e, k_{u_{1}}}^{2}=0.906 \quad, \quad r_{\varepsilon, k x_{2}}^{2}=0.907$, $r_{\varepsilon, k x_{1}}^{2}=0.914 \quad, \quad r_{\varepsilon, k_{x 3}}^{2}=0.934 \quad$. Corresponding structures on which the theorem 3 is based, are shown on fig. 1. Corresponding structures on which the theorem 3 is based, are shown on fig. 1 .

On fig. 2 show variables $y_{n}, \varepsilon_{n}$. They confirm presence at system of the distributed lag on $u_{1, n}$. As inputs are random calculate criterion $d$. Receive $d=0.54$ that following[2], confirms result about presence a lag.

Consider system (1) with $A=[0.5 ; 1.4 ; 1.7]^{T}, \quad B=0.6$, $X_{n}=x_{n}=y_{n-1}$. Receive indicator $\Delta(\Psi)=0.03$. Make a solution on presence multicollinearity (nonlinearity) in systems. Apply algorith $\mathrm{m} C D_{\rho}$. Receive $x_{n}=y_{n-1}$. The length of a lag is equal 1. Generate variable $\varepsilon_{n}$ on the basis of a method described in section 5. On fig. 3 show variables $y_{n}, \varepsilon_{n}$ to estimate delay presence on $y_{n-1}$.

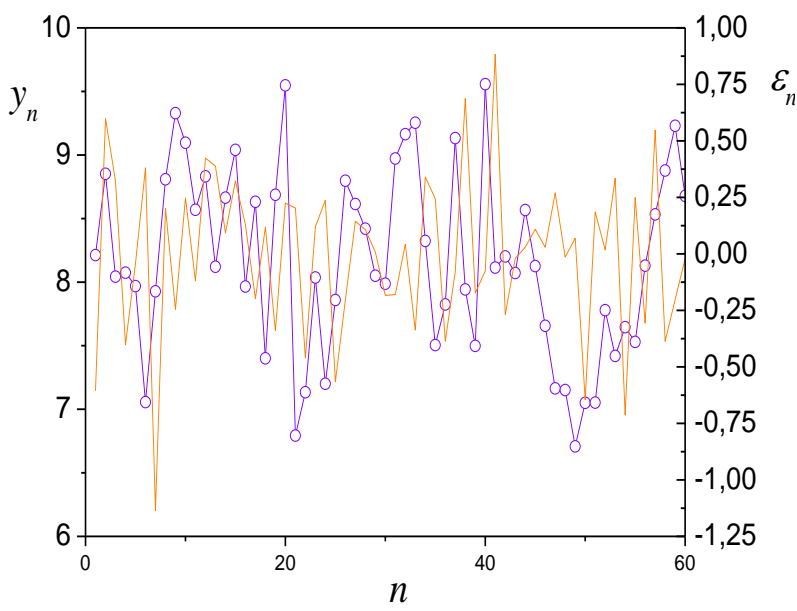

Figure 3. Delay in system (1) with $X_{n}=y_{n-1}$

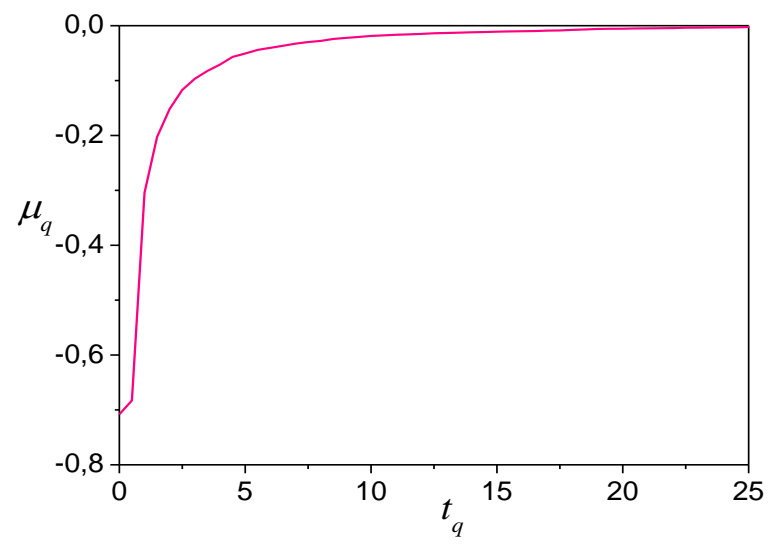

Figure 4. Lyapunov characteristic index $\mu$ 
For validation of the received conclusion estimate CSP $k_{s, x_{i}, e, n}$ at $X_{n}=y_{n-1}$, using dependences (17), (18) and (19). Problem reduce to estimation $\lambda$ in (17). On fig. 4 show change $\mu_{n}$ on the basis of (19). Suppose $\hat{\lambda}=-0.008$. More exact estimation $\lambda$ in (17) define, using a method offered in[24]. Receive $\hat{\lambda}=-0.0102$.

\section{Conclusions}

The is functional-multiple approach to structural identification of static systems with the distributed lag is offered. Solution about structure of the distributed lag of system receive on the bas is of the analysis of special static structures. The criterion of an estimation of degree of linearity of system in parametrical space is introduced. It is considered variants of identification of system in the availability of a lag on dependent and independent variables. The approach to an

\section{REFERENCES}

[1] Malinvaud, E., Statistical methods in econometrics, 3d ed., North-Holland Publishing Co, Amsterdam, 1980.

[2] Johnston J., Econometric methods. 2nd ed., McGraw-Hill Book Company, New York, 1972.

[3] Demetriou I. C., Vassiliou E. E., “An algorithm for distributed lag estimation subject to piecewise monotonic coefficients", International Journal of Applied Mathematics, vol. 39 , no. 1 , pp. 1-10. 2009.

[4] Dhry mes, P.J., Distributed Lags: Problems of Estimation and Formulation, Holden-Day, San Francisco, 1971.

[5] Gershenfeld, N., The Nature of Mathematical Modelling. Cambridge University Press, Cambridge, 1999.

[6] Kailath, T. (ed.), Linear Least-Squares Estimation, Dowden, Hutchinson and Ross, Inc., Stroudsburg, Pennsylvania, Benchmark Papers in Electrical Engineering and Computer Science, v. 17, 1977.

[7] Karabutov, N.N., Structural identification of systems: the analysis of informational structures, URSS, Librokom, Moscow, 2009. (in Russia).

[8] Armstrong, B., "Models for the relationship between ambient temperature and daily mortality" Epidemiology, vol. 17(6), pp. 624-631, 2006.

[9] Nelson, C. R. Schwert, G.W., "Estimating the Parameters of a Distributed Lag Model from Cross-Section Data: The Case of
Hospital Admissions and Discharges", Journal of the American Statistical Association., vol. 69, no. 347, pp. 627-633, 1974

[10] Gasparrini A., Armstrong B., Kenwardb M.G., "Distributed lag non-linear models", Statistics in Medicine, vol. 29(21), pp. 2224-2234, 2010.

[11] Fisher I., "Note on a Short-cut Method for Calculating Distributed Lags", Bulletin de l'Institut International de Statistique, vol. 29, 1937.

[12] Коуск L. M., Distributed Lags and Investment Analysis, North-Holland Publishing Company, 1954.

[13] Almon S., "The distributed lag between capital appropriations and expenditures", Econometrica, vol. 33, pp. 178-196, 1965.

[14] Theil H., Stern R. M., "A simple unimodal lag distribution", Metroeconomica, vol. 12, pp. 111-119. 1960.

[15] Solow R., "On a family of lag distributions", Econometrica, vol. 28, pp. 393-406, 1960.

[16] Jorgenson D.W., "Minimum variance, linear, unbiased seasonal adjustment of economic time series", Journal of the American Statistical Association, vol. 59, no. 307, pp. 681-724, 1964.

[17] Demetriou I. C., E. E. Vassiliou, “A distributed lag estimator with piecewise monotonic coefficients" in Proceedings of the World Congress on Engineering, vol. 2, WCE 2008, July 2 - 4, London, U.K, 2008.

[18] Yoder J., "Autoregressive distributed lag models", WSU Econometrics II, pp. 91-115, 2007.

[19] Cheng Hsiao. Analy sis of Panel Data, Cambridge University Press, 2003.

[20] Wen-Jen Tsay, The Long Memory Autoregressive Distributed Lag Model and Its Application on Con gressional Approval, Institute of Economics, Academia Sinica, 2005.

[21] Carter R. A. L., Zellner A., The ar ar error model for univariate time series and distributed lag models, http://faculty .chicagobooth.edu/arnold.zellner/more/CURRE NT-PAPERS/ararerrm.pdf

[22] Lansing K. J., "Real-Time Estimation of Trend Output and the Illusion of Interest Rate Smoothing", FRBSF Economic Review, pp. 18-34, 2002.

[23] Duffee Gr. R., Term structure estimation without using latent factors, Haas School of Business University of California Berkeley, 2005.

[24] Karabutov, N.N., Structural identification of static plants: Fields, structures, methods, URSS, Librokom, Moscow, 2011. (in Russia).

[25] Lyapunov, A.M., General problem about a movement stability, State publishing house of the techniko-theoretical literature, Moscow, 1950. 